\title{
DOCUMENTOS DEL MUSEO NACIONAL DE CIENCIAS NATURALES DE MADRID, REFERENTES A LAS CÁTEDRAS DE BOTÁNICA DEL REAL JARDÍN BOTÁNICO
}

\author{
José Luis Maldonado Polo \\ Dpto. de H.a de la Ciencia - Centro de Estudios Históricos, CSIC - C/ Duque de Medinaceli 6, Madrid
}

\section{RESUMEN}

Como parte sustancial del proyecto de investigación La Historia Natural en Madrid al final del Antiguo Régimen (CAM, ${ }^{\circ}$ 180/92), se ha realizado el estudio de los fondos manuscritos e impresos existentes en el Museo Nacional de Ciencias Naturales de Madrid (MNCN), pertenecientes o relativos al Real Jardín Botánico de Madrid (RJB). Los materiales se han indizado, ordenado y clasificado, con el resultado que presentamos a continuación.

\section{SUMMARY}

As an essential part of the research project The Natural History in Madrid at the end of the Old Regime (CAM, n 180/ 92), has been carried out the study of the manuscripts and printed funds existent in the National Museum of Natural Sciences from Madrid (MNCN), belonging or relative to the Royal Botanical Garden from Madrid (RJB). ${ }^{\circ}$ The stuff have been ordered and classified, with the outcome which we presented next.

Dentro del proyecto de investigación La Historia Natural en Madrid al final del Antiguo Régimen, financiado por la Comunidad Autónoma de Madrid (Rf. $\mathrm{n}^{\circ}$ 180/92), en el año de 1994, hemos realizado en primer lugar la prospeción, búsqueda y evaluación de los fondos documentales, tanto manuscritos como impresos, del material existente en el Archivo del Museo de Ciencias Naturales, pertenecientes al Real Jardín Botánico de Madrid (RJB) o relacionados con esta institución.

A continuación se ha realizado un índice general, ordenando y clasificando los documentos por materias, disponiéndolos físicamente en cajas y manteniendo, dentro de lo posible, la numeración existente hasta el momento. De esta manera el total de la documentación ha quedado clasificada de la forma siguiente: 


\section{JOSÉ LUIS MALDONADO POLO}

* Cajas 23, 32 y 32 bis

- Cuentas de gastos: obras, materiales, jornales, nóminas, listas, recibos..., en los años 17861792, 1799-1803.

* Caja 31 (miscelánea)

- «Relación de contenidos de varios cajones (?)». Estos cajones a los que se refiere el título original son; 1, 4-10, 12, 24-32 [finales del siglo XIX].

- Sobre la Flora Española, años: 1775, 1784, 1786-1793.

- Sobre la «Ceres Española» y otras cuestiones botánicas de 1827-1830, 1836 y 1871.

* Caja 33 (miscelánea)

- Carta de Francisco A. Rico a José Quer, (1758).

- Reglamento del RJB, (1783).

- Nuevo Reglamento del RJB y nombramiento de Cavanilles como director, 17 de junio de 1801 .

- Edicto de oposición de Botánico de Cámara de $3^{a}$ clase de la Real Botica, (1807).

- Reglamento interno para el RJB, [1817], dos copias.

- Reglamento del RJB, 12 de agosto de 1817.

- Proyecto de Reglamento Económico para el RJB, (1818).

- Colección de edictos (duplicados) para la oposición a la plaza de viceprofesor de botánica, (1832).

- Sobre leyes y ordenanzas de montes, 1836-1837

- Relaciones de documentos del Archivo del RJB, realizado por Mariano Lagasca, (1857).

- Acerca de la Real Academia de Ciencias, 1856-1858

.Escuela de Agricultura y Asociación de Agricultores de España, [1834, 1835, 1881-1883].

* Caja 34 (miscelánea)

- «Flore D`Egypte. Descriptions des plantes graves...», por Mr. Delile a Lagasca, (1834).

- Herbario de Lagasca, (1800, 1861-1863).

- Inventarios de maderas, resinas y frutos que existen en el Gabinete de Historia Natural de Madrid y que se trasladan al RJB, (1844).

- Herbario de Demetrio Rodríguez, (1847).

* Cajas 35-39,41-55

Documentos relativos a cuestiones administrativas y de régimen interno del RJB: personal, correspondencia, cuentas, sueldos, librería (biblioteca), corresponsales, obras, remisión de semillas, plantíos, siembras, nombramientos, listas de empleados, etc., (años, 1788, 1793, 1796, 1797, 1801-1805, 1812, 1814-1865, 1868-1905, 1920-1928, 1934, 1937 y 1938). Pertenecientes a esta serie de cajas, señalamos a continuación algunos documentos singulares:

Caja 37, años 1818-1823, documentos referentes a la huerta del convento de los Jerónimos para ubicar la Escuela de Agricultura. En esta misma caja y en los años 1818 y 1819 documentos referentes a los arreglos de la estufa y cañería.

Caja 39, año 1827, «Disertación...» de Graells.

Caja 41, año 1829, folletito impreso con «Observaciones sobre el artículo de la cria de la cochinilla en Málaga...».

Caja 44, dos folletos impresos: «Contestación a la Filología homeopática. Por el Dr. Juan Sanlleghi», (sin fecha) y «Cuestión acerca del establecimiento de una clínica homeopática...», 1848. 


\section{DOCUMENTOS DEL MUSEO NACIONAL DE CIENCIAS NATURALES DE MADRID}

Caja 46, año 1857, sobre la construcción de la estufa caliente e «Ynventario del material del Jardín Botánico».

Caja 50, año 1863, extenso escrito de Mariano de la Paz Graells sobre la historia del RJB.

Caja 51, año 1864, folletito impreso «El Jardín Botánico y Zoológico de Madrid», por M. de la P. Graells y de 1865 , otro folletito «Index seminum...».

Caja 52, permisos de salida de objetos del RJB, años 1857-1865.

Caja 53, año 1871, varios documentos sobre el herbario de Isern.

Caja 54, año 1893 y 94, sobre el cultivo del tabaco.

Caja 55, año 1938, herbario y biblioteca de Carlos Pau.

Finalmente, dentro de estos fondos referentes al RJB, hemos apartado la documentación referente a las Cátedras de Botánica Médica, Agrícola y General, agrupándola en cajas con numeración independiente, por tratarse de un tema específico y separado del resto de la documentación de la institución botánica. Las cajas con su contenido han quedado agrupadas de la forma siguiente:

* Caja ${ }^{\circ} 1$. Matrícula y listado de alumnos, con el contenido y años siguientes:

- Botánica médica, matrícula de alumnos procedentes de los ramos de medicina, farmacia y cirugía, así como otros aficionados a esta disciplina científica. Años 1788-1803, 1815, 1833 y 1845. Además, del año 1789 existe una lista de profesionales sanitarios que han dado lecciones desde el 3 de abril hasta el 18 de julio y otra lista de discípulos de botánica que se presentaron a oposiciones en ese mismo año. De 1794, hay una lista de alumnos de veterinaria que asistieron a clases de botánica.

- Botánica agrícola, matricula de alumnos, años 1810, 1814, 1816,

1817, 1820-1822, 1829, 1830, 1832-1837, 1845. Lista de alumnos examinados de 1836.

- Botánica General, matricula y lista de alumnos de los años 1816-1823, 1825-1843.

* Caja ${ }^{\circ} 2$. Contiene toda la documentación de la Cátedra de Botánica Agrícola o Agricultura, reunidos en expedientes, dispuestos por orden cronológico de los años, 1815-1820, 1822, $1824,1827-1838,1840,1843,1848,1854$. En el catálogo que se ha confeccionado se indican la fecha y localidad de emisión de los principales documentos, así como de aquellos que complementan cada expediente, y el tipo documental de cada uno. Además, se hace una descripción del contenido, se señala el $n^{\circ}$ de hojas, y su formato.

* Caja $n^{\circ} 3$. Incluye documentos relativos a cuestiones generales de las tres cátedras, y otros sobre materias específicas de las cátedras de Botánica Médica y la de Organografía y Fisiología Vegetal.

* Caja nº 4 . Contiene la documentación íntegra de la Cátedra de Botánica General o Sistemática.

Finalmente, hemos de advertir que estas dos últimas cajas han quedado pendientes de su catalogación. Tarea que esperamos acometer más adelante, siguiendo los criterios utilizados ahora para la catalogación de la caja $\mathrm{n}^{\circ} 2$, y completar así el catálogo del fondo documental relativos al RJB existente en el archivo del MNCN.

A continuación presentamos el catálogo correspondiente a la Cátedra de Botánica Agrícola referidol.

1 Agradezco a Manuel Parejo la ayuda prestada en la localización del material documental y en la mecanografía del propio catálogo.

Asclepio-Vol. XLVIII-1-1996 


\section{JOSÉ LUIS MALDONADO POLO}

\section{CATÁLOGO DE LOS DOCUMENTOS DE LA CÁTEDRA DE BOTÁNICA AGRÍCOLA}

\section{CAJA 2}

- 1815, febrero 17, Madrid.

«Plan o Método de enseñanza agrícola que deberá seguir en las 6 cátedras o escuelas de Agricultura mandadas establecer en el Reyno», a propuesta de la Real Sociedad Económica Matritense (RSEM), por Antonio Sandalio de Arias, Simón de Rojas Clemente y Claudio Boutelou. 8 h., f.

- 1815, noviembre 28, Madrid.

Certificado de Antonio Osteret y Navío a favor de A. Sandalio Arias como opositor al premio de una medalla de oro por confeccionar una cartilla elemental de Agricultura y otros méritos de este socio de la RSEM. 4 h., f.

- 1815, noviembre 30, Madrid.

Relación de Mariano Lagasca sobre los méritos y servicios de Antonio Sandalio. 4 h., f.

- 1815, diciembre 22, Madrid.

Carta de agradecimiento de Antonio Sandalio al Marqués de Santa Cruz por su nombramiento como Profesor de Agricultura del Real Jardín Botánico (RJB). 2 h.

- 1816, enero 1, Madrid.

Carta de A. Sandalio al Marqués de Santa Cruz proponiendo el inicio del curso de Agricultura en el próximo mes de Febrero. 2 h. Corren unidos: visto bueno de la Junta de Protección de Ciencias Naturales (JPCN), sobre la propuesta de Sandalio. 2 h. Contiene: oficio de este mismo dirigido a Antonio Gutiérrez avisando del comienzo de las clases de Agricultura el 3 de febrero y continuando los lunes, miércoles y viernes de cada semana. Madrid, 25. 2 h. Nota informativa sobre estas fechas. 2 h. Avisos de inclusión de esta apertura en la próxima «Gaceta». Madrid, 28. $5 \mathrm{~h}$.

- 1816, septiembre 5, Madrid.

Informe negativo de A. Sandalio sobre el manuscrito de Juan García Navarro «Reflexiones sobre la Agricultura...». 40 h. Se adjunta el citado manuscrito. 39 h., +2 tapas y minuta de la Junta de Protección de Ciencias Naturales sobre la recepción de 5 ejemplares de la disertación, así como por el informe de Sandalio. $2 \mathrm{~h}$.

- 1816, septiembre 26, Madrid.

Oficio de A. Sandalio sobre la instrucción que le solicitan acerca de los objetos necesarios para la Cátedra de Agricultura. $6 \mathrm{~h}$.

- 1816, octubre 3, Madrid.

Oficio de Mariano Lagasca remitiendo el Plan de oposiciones a premios de la Cátedra de Agricultura. 2 h., f. 


\section{DOCUMENTOS DEL MUSEO NACIONAL DE CIENCIAS NATURALES DE MADRID}

- 1817, enero 31, Madrid.

Oficio de A. Sandalio sobre la apertura del curso de Agricultura que señala para el día 8 de febrero. $2 \mathrm{~h}$. Minuta sobre esta fecha y requisitos para la matrícula de los aspirantes. $1 \mathrm{~h}$.

- 1817, julio 28, Madrid.

Oficio de A. Sandalio remitiendo el «Memorial» para enviarlo al rey y otras cuestiones referentes a la escasa dotación de medios para la plaza de profesores de Agricultura. $2 \mathrm{~h}$.

- 1817, agosto 4, Madrid.

Oficio de la JPMNCN sobre la representación de A. Sandalio acerca de su petición de alguna ayuda para la Cátedra. $2 \mathrm{~h}$.

- 1817, agosto 14, Madrid

Oficio de A. Sandalio sobre la falta de tiempo que tiene para la Cátedra de Agricultura para realizar a la vez las tareas del cargo de Jardinero mayor, por lo que solicita se le libere de este último trabajo. $4 \mathrm{~h}$.

- 1817, agosto 15, Madrid.

Oficio de M. Lagasca al Marqués de Santa Cruz informándole sobre los acuerdos de la $1^{\mathrm{a}}$ sesión de la Junta Directiva y económica del Real Jardín Botánico referentes a los cargos de A. Sandalio. $2 \mathrm{~h}$.

- 1818, febrero 12, Madrid.

Oficio de M. Lagasca al Marqués de Santa Cruz sobre el comienzo del curso de Agricultura para el 21 del mes en curso y los días en que deberán continuar las clases. 2 h., f.

- 1818, diciembre, Madrid.

Minuta para la convocatoria al concurso de opositores a las seis Cátedras de Agricultura. 2 h., f.

- 1819, marzo 1, Madrid.

Oficio de José Elizondo al Presidente de la Junta de Protección del Museo Nacional de Ciencias Naturales (JPMNCN) sobre las oposiciones a las Cátedras de Agricultura de Sevilla, Valencia, León, Burgos, Badajoz y Toledo y los individuos que deben componer los tribunales, con especial referencia a Antonio Sandalio. 2 h. Corren unidos: oficios de Antonio Gutiérrez sobre la pretensión de A. Sandalio de suspender las clases para poder formar parte del Tribunal de oposiciones. Madrid, 6 de marzo. 4 h. Oficio de José Elizondo sobre la carencia de la RSEM de local para realizar los ejercicios prácticos de las seis Cátedras. Madrid, 25 de marzo. $2 \mathrm{~h}$. Oficio sobre la realización de esos ejercicios en el Real Jardín Botánico, Madrid, 29 de marzo. $2 \mathrm{~h}$.

-1819 , junio 2, Madrid.

Nota informativa de Alejandro Olivan (impresa), sobre lo que el Marqués de Casa Irujo trasmitió a la RSEM, acerca de la enseñanza de Agricultura y de la obra de A. Sandalio: «Lecciones de Agricultura». $2 \mathrm{~h}$. 


\section{JOSÉ LUIS MALDONADO POLO}

- 1819, diciembre 24, Madrid.

Informe de A. Sandalio a la Junta Directiva del Real Jardín Botánico exponiendo los motivos para el cambio de fecha de apertura del curso de Agricultura. $4 \mathrm{~h}$. Se incluye: oficio de Lagasca al Marqués de Santa Cruz enviándole el anterior informe. Madrid, 30. 2 h. Oficio de Lagasca sobre el acuerdo de la Junta de Protección del Museo. Madrid, 1 de enero 1820. 2 h.

$-[1820]$, enero 7.

Minuta de Antonio Sandalio sobre el comienzo del curso de Agricultura en el Real Jardín Botánico. 1 h.

- 1820, agosto 13, Madrid.

Oficio de M. Lagasca sobre las certificaciones impresas que se dan a los alumnos de Agricultura. 2 h. Oficio de A. Sandalio al Presidente de la Junta de Protección sobre la nueva forma de expedir certificados a los alumnos. Madrid, 14.2 h.

- 1820, diciembre 2, Madrid.

Oficio de M. Lagasca al Presidente de la JPMNCN, acerca de la proposición de explicar lecciones de Agricultura en la Sala de Mineralogía y las fechas señaladas para esta enseñanza. $2 \mathrm{~h}$. Contiene: minutas acerca de estos pormenores. $2 \mathrm{~h}$. Borrador dirigido al Presidente del Real Jardín Botánico sobre estos mismos puntos. [Madrid], 29 de diciembre [1820]. 1 h. Oficio de M. Lagasca al Secretario de la Junta de Protección sobre estas consideraciones. Madrid, 30. 2 h.

- 1822, enero 24, Valencia

Certificado de Joaquín Piñol de la liquidación de cuentas de la Cátedra de Agricultura desde el 10 de mayo de 1819 a diciembre de 1821.2 h., f.

- 1824, enero 14, Madrid.

Oficio de Antonio Gutiérrez al Primer Secretario de Estado sobre la propuesta de Antonio Sandalio acerca de comenzar las clases de Agricultura en el Real Jardín Botánico. 2 h. También: borrador de la decisión real sobre la continuidad de la enseñanza de Mineralogía y Zoología en las cátedras del Gabinete de Historia Natural (GHN) y las fechas de comienzo de éstas. [s.f]. 1 h.

- 1827, mayo 31, Madrid.

Informe de José Demetrio Rodríguez a Jacobo $\mathrm{M}^{\mathrm{a}}$ de Parga sobre la solicitud de José Alonso Quintanilla relativa a la opción de acceder a la Cátedra de Agricultura. 4 h. Corren unidos: oficio de remisión de Manuel González Salmón al Presidente de la JPMNCN de una instancia de J.A. Quintanilla solicitando la plaza vacante. Nota marginal en la que se aprueba la indecisión de Quintanilla, como opositor. Madrid, 18 de noviembre. 2 h. Oficio al Primer Secretario de Estado proponiendo a Quintanilla como sujeto idóneo a la plaza vacante. Madrid, 29 de noviembre. $1 \mathrm{~h}$. 


\section{DOCUMENTOS DEL MUSEO NACIONAL DE CIENCIAS NATURALES DE MADRID}

- 1828 , enero 22, Palacio.

Comunicación de Manuel González Salmón al Presidente de la JPMNCN, sobre la decisión real denegando la solicitud de Quintanilla, aceptando, por otro lado, que éste pueda presentarse a la oposiciones para profesores de Agricultura, cuando se celebren. $2 \mathrm{~h}$.

- 1828, febrero 8, Madrid.

Edicto para la oposición a la Cátedra de Agricultura y plaza de Jardinero Mayor del Real Jardín Botánico, firmado por Vicente Soriano. 2 h., f. Se adjuntan: 2 borradores del mismo. 3 h., f. Oficio de remisión de el Conde de Argüello al Secretario de la JPMNCN del plan de oposiciones y edicto para la plaza vacante así como una solicitud de Jorge Checa. Nota marginal. Madrid, 10.2 h. Borradores de oficios dirigidos al Conde de Argüello sobre la difusión del edicto de convocatoria de oposición tanto para los opositores como a los examinadores. Madrid, 29. 2 h.

- 1828, febrero 11, Madrid.

Minuta del Plan de oposiciones a la Cátedra de Agricultura y Jardinero Mayor del Real Jardín Botánico. 2 h., f.

- 1828, marzo 10, Palacio.

Oficio de remisión de M. González Salmón al Presidente de la JPMNCN de una instancia de A. Sandalio para concurrir a la oposición de la Cátedra de Agricultura. 2 h. Borrador de oficio dirigido al Primer Secretario de Estado sobre la citada instancia de Sandalio en la que solicita ser restituido en la Cátedra o al menos ser incluido en la oposición convocada. Madrid, 9 de abril. $1 \mathrm{~h}$.

- [1828], Madrid.

Oficio de A. Sandalio al Conde de Argüello remitiéndole tres minutas del anuncio que deberá publicarse en la «Gaceta y Diario» sobre la oferta de la Cátedra de Agricultura. Se incluyen las 3 minutas. $2 \mathrm{~h}$.

- 1828, Junio 3, Palacio.

Oficio de M. González Salmón al Presidente de la JPMNCN comunicándole la decisión del rey acerca del nombramiento de A. Sandalio como Catedrático de Agricultura y Jardinero Mayor del Real Jardín Botánico. 2 h. Corren unidos: minuta dirigida al Conde de Argüello transmitiéndole el nombramiento. Madrid, 12. 1 h. Oficio del Conde de Argüello a Ramón Garcés de Marcilla informándole de dar cumplimiento a la orden real sobre el nombramiento de Sandalio. Madrid, 15. 2 h. Minutas dirigidas al Conde de Argüello y al Primer Secretario de Estado anunciándole la toma de posesión de A. Sandalio y la apertura del Curso. Madrid, 25 de junio y 10 de julio. $2 \mathrm{~h}$

- 1828, septiembre 18, Madrid.

Oficio de A. Sandalio al Conde de Argüello sobre la posibilidad de examinar y conceder los premios correspondientes a los alumnos de botánica ante la conclusión de las clases. Se incluye: nota marginal de 24 de ese mes que contiene la negativa de efectuar exámenes y conceder los premios. 2 h. Minuta dirigida a A. Sandalio dándole cuenta del oficio anterior. Madrid, 24. $1 \mathrm{~h}$. 


\section{JOSÉ LUIS MALDONADO POLO}

- 1828, septiembre 25, Madrid.

Oficio de A. Sandalio a Ramón Garcés de Marcilla, sobre lo inadecuado que es negar el examen a los alumnos matriculados en el curso y expone las razones. $2 \mathrm{~h}$. Se incluye la minuta a A. Sandalio rectificando la decisión de la JPMCN y aceptando su propuesta. Madrid, 26. $1 \mathrm{~h}$.

- 1828, octubre 7, Madrid.

Oficio de A. Sandalio a R. Garcés de Marcilla, remitiéndole la lista de los alumnos de Agricultura que se han presentado al examen. $2 \mathrm{~h}$. Corren unidos: minutas a A. Sandalio remitiéndole la orden de la JPMNCN con las 9 certificaciones solicitadas para los alumnos aprobados y expresándole que debe incluir en ella el tiempo de duración del curso que ha finalizado. Madrid, 17 y 18.2 h. Lista de los alumnos examinados. 2 h., f. Oficio de A. Sandalio a R. Garcés remitiéndole las certificaciones de los alumnos aprobados e insistiendo en la validez de ellas por lo adecuado del curso impartido. Madrid, 20. 2 h. Minuta a A. Sandalio comunicándole la negativa de la JPMNCN acerca de la indecisión en los certificados del tiempo de duración del curso. Madrid, 25. $1 \mathrm{~h}$. Minuta a A. Sandalio devolviéndole los nueve certificados firmados de los alumnos aprobados en el curso. Madrid, 11 de noviembre. $1 \mathrm{~h}$.

- 1828, octubre 25, Madrid.

Minuta al Conde de Argüello negando la reparación que necesite la «Estufa del Mediodía» del Real Jardín Botánico por problemas económicos. 1 h.

- 1828, octubre 31, Madrid.

Minuta al Primer Secretario de Estado, transmitiéndole el anuncio de apertura del Curso de Agricultura. $1 \mathrm{~h}$.

- 1828, diciembre 19, Madrid.

Oficio de A. Sandalio al Conde de Argüello adjuntándole la minuta con las horas y los días en que debe comenzar el curso de Agricultura. $2 \mathrm{~h}$. Se acepta la citada minuta. $1 \mathrm{~h}$.

-1829 , diciembre 21, Madrid.

Oficio del Conde de Argüello a Ramón Garcés referente a la compra de plata procedente de Francia y a la apertura del curso de Agricultura. Asimismo, indica el número y precio de árboles y arbustos que se plantarán en el Real Jardín Botánico. 2 h.

- 1829, junio 15, Madrid.

Oficio del Conde de Argüello a la JPMNCN señalando que este día ha terminado el curso de Agricultura y que el 17 comenzaran los exámenes. $2 \mathrm{~h}$.

- 1829, junio 20, Madrid.

Oficio de Antonio Sandalio al Conde de Argüello, remitiendo la lista de alumnos del Curso de Agricultura, aprobados en los exámenes celebrado el día 17. 2 h. Se adjuntan: la lista. 1 h., f. Oficio del Conde de Argüello a Ramón Garcés, remitiéndole el oficio de Antonio Sandalio sobre este particular. Nota marginal con la decisión de la Junta. Madrid, 21 y 24.2 h. Minuta a Antonio Sandalio remitiéndole el acuerdo de la JPMNCN Madrid, 24. 1 h. Oficio de Antonio Sandalio a Ramón Garcés dándole los 11 certificados de los alumnos de Agricultura. Madrid, 25. 2 h. Minuta a Antonio Sandalio sobre el Oficio de 25 de julio. Madrid, 12 de julio. $1 \mathrm{~h}$. 
- 1829, julio 17, Palacio.

Oficio de M. González Salmón al Presidente de la JPMCN sobre la proposición de supresión de 2 cátedras de Agricultura, en Badajoz y en Llerena. 2 h. Minuta dirigida al Secretario de Estado con la decisión de la Junta sobre el particular. Madrid, 2 de agosto. 1 h. Oficio de M. González Salmón al Presidente de la JPMNCN con la decisión del rey. Madrid, 21. 2 h. Borrador dirigido al Primer Secretario de Estado sobre las ventajas de esta enseñanza de la Agricultura en las Cátedras. Madrid, 30 de septiembre. 2 h. Oficio de M. González Salmón al Presidente de la JPMNCN informando del acuerdo de susistución de la expresadas Cátedras. Madrid, 27 de febrero de 1830. $2 \mathrm{~h}$.

- 1829, diciembre 24, Madrid.

Oficio de Antonio Sandalio a Pedro Alcantara señalando la fecha de comienzo del curso de Agricultura y remitiendo la minuta para su publicación en la «Gaceta de Gobierno» y en el «Diario de Avisos de esa Corte». 2 h. Se incluye: la citada minuta y otra con distinta fecha de comienzo. 2 h. Otras 2 minutas dirigidas a la JPMCN y al Primer Secretario de Estado. Madrid, 28 y $31.3 \mathrm{~h}$

- 1830, junio 27, Madrid.

Oficio de Antonio Sandalio a Ramón Garcés devolviendo las nuevas certificaciones de los alumnos aprobados en la Cátedra de Agricultura. 2 h. Acompaña un oficio de Pedro Alcantara al Secretario de la JPMCN remitiendo la lista de los alumnos aprobados. Madrid, 19. Nota marginal con la decisión de la sesión de la Junta del día 21. 2 h. Minuta a Antonio Sandalio remitiendo nuevas certificaciones en blanco para rellenarlas. Madrid, 26. $2 \mathrm{~h}$.

- 1830, septiembre 30, Palacio.

Oficio de M. González Salmón al Presidente de la JPMNCN informando de lo señalado al asistente de Sevilla sobre reemplazar a José Lucio Pérez para Claudio Boutelou como Profesor de Agricultura. $2 \mathrm{~h}$.

-1830 , diciembre 1.

Carta de Antonio Sandalio al Conde de Argüello remitiendo el Reglamento y convocatoria de oposición a la Cátedra 2 h. Se incluye el citado reglamento. 2 h., f.

-1830 , diciembre 26.

Oficio del Conde de Argüello a Ramón Garcés remitiéndole el anuncio de comienzo del Curso de Agricultura. 2 h. Minuta al Primer Secretario de Estado sobre el anuncio. Madrid, 31. 2 h.

- 1830, diciembre 31, Madrid.

Minuta dirigida al Primer Secretario de Estado sobre la solicitud de Pascual Asensio de cambiar su plaza como profesor de Agricultura de Burgos, por la de Valencia. 1 h. Oficio de Manuel González Salmón a Diego Clemencin adjuntandole la orden real sobre el restablecimiento de la Cátedra de Agricultura de Valencia. Madrid, 25 de noviembre. 2 h. Minuta al Primer Secretario de estado sobre estos pormenores. Madrid, 8 de diciembre. 1 h. Oficio de M. González Salmón a Diego Clemencin sobre la permuta de Pascual Asensio. Palacio, 20. 2 h. 


\section{JOSÉ LUIS MALDONADO POLO}

- 1831, enero 12. Palacio.

Oficio de M. González Salmón al Presidente de la JPMNCN sobre lo comunicado al Secretario de la Sociedad Económica de Valencia, del nombramiento de Pascual Asensio como Profesor de Agricultura. $2 \mathrm{~h}$.

- 1831, febrero 20, Palacio.

Oficio de M. González Salmón al Presidente de la JPMNCN sobre la decisión real de que el Catedrático de Agricultura del Real Jardín Botánico y demás Jardineros del Buen Retiro y Moncloa acudan a una posada para conversar con el «Director de Arbolados del Real Canal de Aragón», Francisco Sanguesa sobre el fomento del arbolado. $2 \mathrm{~h}$.

- 1831, junio 20, Madrid.

Lista firmada por Antonio Sandalio de los alumnos de Agricultura aprobados en el Curso. $2 \mathrm{~h}$.

- 1831, agosto 29, Madrid.

Oficio de Pedro Alcantara a Ramón Garcés sobre la solicitud que hace el Jardinero Mayor [A. Sandalio] sobre aumento de sueldo ya que el que cobra en la actualidad le es insuficiente. Acompaña: nota marginal de 31 de septiembre de la JPMNCN, que incluye la decisión de aumento a 18.000 reales. 2 h. Minuta al Primer Secretario de Estado sobre la petición de Antonio Sandalio para sus comisiones profesionales. Madrid, 3. $1 \mathrm{~h}$.

- 1831, diciembre 30, Madrid.

Oficio de Antonio Sandalio recordando las fechas de apertura del Curso de Agricultura. Nota marginal con la resolución de la JPMNCN de 2 de enero de 1832. 2 h. Minutas sobre estas fechas. Madrid 22 de enero de 1832. $2 \mathrm{~h}$.

- 1832, junio 8, Madrid.

Lista de alumnos, firmada por Antonio Sandalio, del Curso de Agricultura que han sido aprobado. 1 h. Oficio de remisión del Conde de Argüello a Ramón Garcés con la lista. Madrid, 12 y $21.3 \mathrm{~h}$.

- 1832, octubre 13, Madrid.

Solicitud de Domingo García de una nueva certificación de haber participado en el curso de Agricultura de 1828-29, por haberse extraviado la que se le dió. 1 h., f. Incluye (por duplicado), la decisión de la JPMNCN ordenando que se le proporcione lo que pide. Madrid, 15. $2 \mathrm{~h}$.

- 1832, diciembre 29, Madrid.

Oficio de Antonio Sandalio al Comisario del Real Jardín Botánico con las fechas del nuevo curso de Agricultura, para su divulgación. 2 h. Corren unidos: oficio del Conde de Argüello a Ramón Garcés con la fecha del 11 de enero para el comienzo del Curso. Madrid, 31. 2 h. Minuta con el anuncio citado. $1 \mathrm{~h}$. Minuta al Secretario de Estado sobre lo mismo. Madrid, 4 de enero de 1833. $1 \mathrm{~h}$. 


\section{DOCUMENTOS DEL MUSEO NACIONAL DE CIENCIAS NATURALES DE MADRID}

- 1833, enero 18, Madrid.

Oficio de Manuel Abad al Secretario de la JPMNCN remitiendo para su censura la «Cartilla elemental de Agricultura» de Antonio Sandalio. 2 h. Acompaña el borrador con la decisión de la Junta. Madrid, 5 de febrero. $1 \mathrm{~h}$.

- 1833, febrero 14, Madrid.

Borrador de Antonio Sandalio remitiendo a la JPMNCN, la obra «El cultivador practico». $1 \mathrm{~h}$. Corre unido: oficio de Antonio Sandalio al Secretario de la JPMNCN, señalando que nada debe impedir su jubilación. Madrid, 17 de marzo. 2 h. Minutas a Manuel Abad informándole sobre el oficio anterior. Madrid, 26 y 19 de octubre. 2 h. Oficio de Antonio Sandalio al Secretario de la JPMNCN devolviéndole la obra para que se dictamine sobre ella. Madrid, 8. 2 h.

- 1833, junio 17, Madrid.

Oficio del Conde de Argüello a Ramón Garcés remitiéndole la matrícula de los alumnos del curso de Agricultura. 2 h. Acompaña: oficio de Antonio Sandalio al Comisario del Real Jardín Botánico, remitiendo la lista de aprobados, en la que incluye a Teodoro Armengaud que no está matriculado en este curso por las razones que él mismo expone en su escrito. Incluye la citada lista. Madrid, 18. 2 h. Carta de Teodoro Armengaud. Madrid, 17. 2 h. Oficio del Conde de Argüello a Ramón Garcés concediendo lo que pide Antonio Sandalio sobre el alumno francés. Madrid, 1 de julio. Nota marginal con el acuerdo de la Junta de 1 de julio. $2 \mathrm{~h}$.

- 1833, julio 15, Madrid.

Borrador dirigido al Secretario de Estado solicitando pensión de viudedad para la esposa e hija de Antonio Sandalio según instancia de éste solicitando al rey la pensión el 10 de julio. $2 \mathrm{~h}$.

- 1833, diciembre 22, Madrid.

Oficio de la JPMNCN, al Fiscal con el nombramiento de Antonio Sandalio como «Instructor general de Montes» debiendo por tanto censar en los demás cargos del Real Jardín Botánico. 2 h. Acompaña la minuta dirigida a Pedro Alcantara sobre este oficio y proponiendo también a Sandalio como miembro de la JPMNCN Madrid, 31. $1 \mathrm{~h}$.

- 1834, enero 24, Madrid.

Oficio de F. Martínez Robles al Secretario de la JPMNCN prestándose para ocupar la Cátedra de Agricultura vacante. $1 \mathrm{~h}$., f.

-1834 , enero 27.

Oficio a la JPMNCN para que informe sobre la incorporación al Real Jardín Botánico de Francisco Martínez Robles en la Cátedra de Agricultura vacante por cese de Antonio Sandalio. 2 h. Acompañan: borradores al Secretario de Estado informando de este asunto así como de las fechas de comienzo del Curso. Madrid, 27 y 30.2 h.

-1834 , febrero 15 .

Oficio del Secretario de Estado con el informe de la Junta sobre la idoneidad de Francisco Martínez Robles para el cargo que se le otorga. $2 \mathrm{~h}$. 


\section{JOSÉ LUIS MALDONADO POLO}

-1834 , abril 17 y 18 , Madrid.

Borradores dirigidos a José Alonso Quintanilla exponiendo el fallecimiento de Francisco Martínez Robles nada más dar comienzo al curso de Agricultura del que fue nombrado Catedrático. 2 h. Corre unido: oficio a la J.P.M.C.N., proponiendo nueva oposición a la plaza vacante por muerte de Martínez Robles. Aranjuez, 14 de mayo. 2 h. Minuta dirigida a Antonio Sandalio y Vicente Soria sobre la resolución de la Junta proponiendo una comisión para resolver la cuestión de la plaza vacante. Madrid, 21. 1 h.

- 1834, mayo 11. Madrid.

Oficio de remisión de J. Alonso Quintanilla a Ramón Garcés de la lista de alumnos matriculados en el curso de Agricultura. $2 \mathrm{~h}$

- 1834, mayo 24. Madrid.

Carta de Isidro Frutos a la JPMNCN, en nombre de Pedro Díaz, comentando que imponderables le impiden matricularse en el curso de Agricultura. 2 h., f. Acompaña: minuta a J. Alonso Quintanilla con el acuerdo de la Junta sobre la inscripción de Pedro Díaz, fuera de plazo, en el curso. Madrid, 27. 1 h. Oficio de J. Alonso Quintanilla a Ramón Garcés con la conformidad de la propuesta de Pedro Díaz y de Pedro Pardavé. Madrid, 29. 2 h. Borrador sobre lo mismo. Madrid, 18. $1 \mathrm{~h}$.

- 1834, mayo 30, Madrid.

Edicto firmado por Antonio Sandalio y V. Soriano, de la JPMNCN, sobre los ejercicios que tendrán que superar opositores a la plaza de Catedrático de Agricultura. 2 h., f. Acompaña: oficio de remisión de Antonio Sandalio al Secretario de la Junta con la minuta del plan de oposición. Madrid, 31. Nota marginal del acuerdo de la Junta con fecha 9 de junio. 2 h. Anuncio para la «Gaceta» del edicto. $1 \mathrm{~h}$. Borrador del oficio dirigido al Secretario de Estado remitiendo el anuncio y el edicto. Madrid, 14. $1 \mathrm{~h}$.

- 1834, junio 22, Madrid.

Oficio a la JPMNCN, sobre la publicación del anuncio convocando la oposición a la Cátedra de Agricultura para el 22 de agosto. 2 h. Acompaña: nota marginal. Minuta a Pedro Alcantara para que se difunda el anuncio a los gobernadores civiles de las provincias. Madrid. 11 de julio. $1 \mathrm{~h}$. Minuta dirigida a uno de los gobernadores. $1 \mathrm{~h}$.

- 1834, julio 2, Madrid.

Oficio de J. Alonso Quintanilla a Ramón Garcés comentando las fechas de finalización del curso de Agricultura y las limitaciones de las clases por fallecimiento de F. Martínez Robles. $2 \mathrm{~h}$.

- 1834, julio 13, Madrid.

Carta de Antonio Sandalio a Ramón Garcés comentándo la mala suerte corrida en la imprenta de la minuta del edicto de convocatoria de la oposición y pide que se saque copia para nuevamente enviarla a la imprenta. $1 \mathrm{~h}$.

- 1834, julio 15, Madrid.

Oficio de Ramón Garcés a J. Alonso Quintanilla con las fechas de comienzo del Curso y las de los exámenes. $1 \mathrm{~h}$. 
DOCUMENTOS DEL MUSEO NACIONAL DE CIENCIAS NATURALES DE MADRID

- 1834, julio 22, Salamanca.

Oficio de José $\mathrm{M}^{\mathrm{a}}$ Cambronero al Presidente de la JPMNCN, sobre la recepción del ejemplar con el edicto de la oposición. $2 \mathrm{~h}$.

- 1834, julio, Madrid.

Convocatoria impresa del edicto de oposiciones a la Cátedra de Agricultura (43 ejemplares). Se agregan 55 oficios destinados a los gobernadores civiles, Madrid 1834. $110 \mathrm{~h}$.

-1834 , julio, 23, 25 y 31 y agosto 1,6 y 8 .

Oficios de los gobernadores civiles de Orense, Santander, Teruel, Cuenca, Murcia, Castellón, Jaén, Lérida, Huelva, Granada, Pontevedra, Barcelona, Ciudad Real, Zaragoza, Valladolid, León, Valencia, Coruña, Lugo, Tarragona, Almería, Sevilla, Málaga, Baleares y Canarias, a Ramón Garcés señalando que han colocado en las ciudades el edicto de la oposición. 52 h.

- 1834, julio 30, Madrid.

Oficio de J. Alonso Quintanilla a Ramón Garcés remitiéndole la lista de los alumnos aprobados en Agricultura. Comenta aspectos de otros alumnos respecto a los exámenes. $2 \mathrm{~h}$. Se incluye: la citada lista. 2 h. Borrador de oficio dirigido a José Alonso Quintanilla comentando la conclusión del curso de Agricultura y felicitándole por su tarea al frente de la Cátedra y le envía los certificados de los exámenes. Madrid, 7 de agosto. 1 h.

- 1834, agosto 19, [Madrid].

Carta de Antonio Sandalio a Ramón Garcés preguntando si pudiera repetir el aviso al Gobernador para que envíe un destacamento al Real Jardín Botánico y le solicita dos ejemplares del edicto de la convocatoria de oposición a la Cátedra. 2 h.

- 1834, agosto 19 y 21 , Madrid.

Solicitudes de Mariano López del Pozo y de Pascual Asensio para que se les incluya entre los opositores a la Cátedra de Agricultura. 4 h. Otra de José Archente. Madrid, 4 de septiembre. 1 h., f.

- 1834, agosto 24, Madrid.

Carta de J. Alonso Quintanilla al Presidente de JPMNCN, exponiendo las circunstancia de su comisión al frente del curso de Agricultura. 2 h., f. Se incluye: borrador sobre lo mismo. Madrid, 13 de septiembre. $1 \mathrm{~h}$. Borrador dirigido a J. Alonso Quintanilla sobre la certificación que corresponde a Francisco Casariego por haber aprobado el curso de Agricultura. 1 h. Oficio de J. Alonso Quintanilla a Ramón Garcés ratificando lo expuesto en el oficio de 24 de agosto. Madrid, 29 de agosto. $2 \mathrm{~h}$.

- 1834, agosto 31, Madrid.

Borrador de oficio dirigido al Secretario de Estado sobre la concesión de una prórroga de la oposición por dificultades en su resolución a causa de la epidemia de cólera. $1 \mathrm{~h}$. 


\section{JOSÉ LUIS MALDONADO POLO}

- 1834, septiembre 8, [Madrid].

Borrador de oficio dirigido a Quintanilla y Soriano acerca de la instancia de P. Asensio sobre que se le nombrase Catedrático de Agricultura sin hacer la oposición. 2 h. Borrador sobre lo mismo. Madrid, 13. $1 \mathrm{~h}$.

- 1834, septiembre 8, Madrid.

Solicitud de José García Sanz para firmar la oposición a la Cátedra de Agricultura, exponiendo sus méritos para ello. Se adjuntan 3 documentos con estos servicios. 4 h., f. También se incluye un oficio del gobernador de Murcia, José Manso y Valiente sobre el manual [escrito por el solicitante] acerca del cultivo de la seda y un Boletín Oficial de Madrid con detalles de este cultivo. Madrid, 22 de mayo y 14 de junio de $1834.4 \mathrm{~h}$.

- 1834, septiembre 22, Madrid.

Oficio de José $M^{a}$ Moscoso de Altamira sobre la denegación por parte de la reina de la petición de P. Asensio de concederle la plaza de Catedrático sin presentarse a la oposición. 2 h.

- 1834, octubre 2, Madrid.

Borrador dirigido a Andrés Alcón del acuerdo de la JPMNCN referente a los opositores a la Cátedra. $1 \mathrm{~h}$.

- 1834, octubre 2, Madrid.

Oficio de Antonio Sandalio a Ramón Garcés sobre su disposición a aceptar el nombramiento como miembro del Tribunal que juzgará la oposición a la Cátedra. $2 \mathrm{~h}$. Se incluye el borrador dirigido a Quintanilla, Arias y Soriano nombrandoles censores de la oposición. Madrid, 29 de septiembre. $2 \mathrm{~h}$.

- 1834, octubre 5, Madrid.

Oficio de José $\mathrm{M}^{\mathrm{a}}$ Moscoso de Altamira nombrando a Antonio Sandalio, Donato García y J. Demétrio Rodríguez censores de la oposición. 2 h. Corre unido: Borrador dirigido al Secretario de Estado sobre lo mismo. Madrid, 4. 1 h. Borrador de oficio a A, Sandalio sobre lo mismo. Madrid, $8.1 \mathrm{~h}$.

- 1834, octubre 11, Madrid.

Oficio de Donato García al Secretario de Estado señalándole su incapacidad para juzgar custiones de agricultura en relación a su nombramiento como censor de la oposición. 2 h., f. Acompaña un oficio de J. M Moscoso de Altamira sobre el mismo asunto. Madrid, 14. $2 \mathrm{~h}$.

- 1834, octubre 14, Madrid.

Oficio de Pascual Asensio sobre las circunstancias de Mariano López del Pozo, Archante, García y él mismo con respecto a la oposición. 2 h.

-1834 , octubre 15 .

Borrador de oficio dirigido a Donato García sobre determinar junto a A. Sandalio y J.D. Rodríguez, lo que sea más conveniente respecto a su incapacidad como censor de la oposición. $1 \mathrm{~h}$. Corre unido el borrador al Secretario de Estado sobre algunos aspectos de la injusticia de Donato García como censor. Madrid, 19.1 h. 
-1834 , octubre 21.

Oficio de J. $\mathrm{M}^{\mathrm{a}}$ Moscoso de Altamira sobre que se amplie el tribunal de oposición con Andrés Alcón y Tomás Villanova sin permitir a ninguno su dimisión en el cargo. 2 h.

-1834 , octubre 21.

Oficio de Donato García a Ramón Garcés sobre el acuerdo tomado por ellos sobre la oposición, quedando él como Secretario de la Junta de Censores. $2 \mathrm{~h}$.

- 1834, octubre 29, [Madrid].

Oficio de A. Sandalio a Ramón Garcés proponiendo el comienzo de la oposición para el 5 de noviembre y no el 3 como estaba previsto. $2 \mathrm{~h}$. Se incluye el borrador del anuncio para el Diario con esta fecha. $1 \mathrm{~h}$.

- 1834, octubre 31, Madrid.

Oficio de Andrés Alcón a Ramón Garcés sobre la constitución de la Junta de Censores de la oposición y el nombramiento de Secretario. $2 \mathrm{~h}$.

- 1834, noviembre 2, Madrid.

Borrador a Antonio Sandalio para que proporcione los gastos para la oposición. $1 \mathrm{~h}$.

- 1834, noviembre 5, Madrid

Oficio de Andrés Alcón a Ramón Garcés sobre remisión de documentos de los opositores. 4 h.

- 1834, noviembre 5, Madrid.

Oficio de Andrés Alcón a Ramón Garcés sobre la sesión de la oposición celebrada ese día en la que intervino José Archente y José García Gómez. Este último propuso retirarse de los ejercicio por carecer del suficiente conocimiento respecto a sus compañeros opositores. $2 \mathrm{~h}$. Incluye el borrador del oficio a A. Alcón con este mismo asunto. Madrid, 6. $1 \mathrm{~h}$.

-1834 , noviembre 5 .

Disertación de José Archente relativo a la oposición. 14 h.

- 1834, noviembre 5, Madrid.

Disertación de Pascual Asensio relativo a la oposición. 16 h.

- 1834, noviembre 21, Madrid.

Oficio de remisión de Andrés Alcón a Ramón Garcés de la censura hecha por el Tribunal de oposición. 2 h. Corre unido: copia de la censura. Madrid, 29. 2 h. Nueva remisión de la censura por parte de Andrés Alcón con algunas correcciones. Madrid, $302 \mathrm{~h}$.

- 1834, noviembre 30, Madrid.

Oficio de J. Ma Moscoso de Altamira, remitiendo un ejemplar de las «Memorias de la Ynstitución Agrónoma de la Habana». 2 h. 


\section{JOSÉ LUIS MALDONADO POLO}

- 1834, diciembre 4, Madrid.

Notificación de Andrés Alcón a Ramón Garcés del acuerdo de la JPMNCN, en las actas de las sesiones celebradas de la Junta de Censuras de la oposición. 2 h. Incluye la notificación dirigida a A. Sandalio y a Andrés Alcón, para que forme parte del Tribunal de oposición a Madrid, 29 de octubre y 27 de noviembre. 2 h.

- 1834, diciembre 5, Madrid.

Oficio de J. Ma Moscoso de Altamira comunicando el nombramiento de Pascual Asensio como Catedrático de Agricultura al haber superado en la oposición al otro opositor. 2 h.

- 1834, diciembre 13, Madrid.

Oficio de Antonio Sandalio al Secretario de la JPMNCN, transmitiendo el oficio de Pascual Asensio sobre su toma de posesión como Catedrático de Agricultura. 2 h.

- 1834, diciembre 15, Madrid.

Oficio de Pascual Asensio a Ramón Garcés preguntando de que fondos habrá de cobrar por su asignación como Catedrático. $2 \mathrm{~h}$.

-1834 .

Expediente de la oposición a la Cátedra de Agricultura. 13 h., f.

- 1835, enero 2, Madrid.

Oficio de J. M Moscoso de Altamaira sobre que se encargue a la Real Sociedad Económica de Madrid el que se trasladen los útiles necesarios del RJB para celebrar la oposición a las Cátedras de Agricultura de Murcia y Santiago. $2 \mathrm{~h}$.

- 1835, febrero 14, Madrid.

Oficio de Pascual Asensio al Secretario de la JPMNCN para que ésta difunda el anuncio de concurso del curso de Agricultura. 2 h. Corre unido: minuta del Secretario de Estado sobre la remisión del anuncio citado. Madrid 16 de febrero. $1 \mathrm{~h}$. Minuta con el anuncio. $1 \mathrm{~h}$.

- 1835, marzo 28, Madrid.

Dos borradores de comunicación a Antonio Sandalio sobre los instrumentos de poda y tala ofrecidos por Salvador López Ramos. $2 \mathrm{~h}$.

- 1835, abril 21, Madrid.

Oficio de remisión de Pascual Asensio a Ramón Garcés con la lista de alumnos matriculados en el curso de Agricultura de este año. $2 \mathrm{~h}$.

- 1835, octubre 3, Madrid.

Comunicación de Pascual Asensio sobre las fechas de los exámenes de Agricultura. 2 h.

- 1835, noviembre 1, Madrid.

Oficio de Pascual Asensio a Antonio Sandalio contestando a su oficio sobre impartir lecciones de horticultura y economía rural y de los medios necesarios para este curso preparatorio. $2 \mathrm{~h}$. 


\section{DOCUMENTOS DEL MUSEO NACIONAL DE CIENCIAS NATURALES DE MADRID}

- 1835, noviembre 23, Madrid.

Oficio de Pascual Asensio a Ramón Garcés sobre los alumnos aprobados en el curso. 2 h. Se adjunta la lista. 1 h., f.

- 1836, febrero 17, Madrid.

Borrador a Pascual Asensio sobre el estado del Real Jardín Botánico en cuanto a sus nuevas enseñanzas y que indique las fechas de apertura del Curso de Agricultura. $1 \mathrm{~h}$. Acompaña: oficio de Pascual Asensio señalando el día 27 para ese inicio. Madrid, 19. 2 h. Minuta al Secretario de Estado del anuncio de apertura. Madrid. 20. 1 h. Oficio de Pascual Asensio a Ramón Garcés sobre la remisión de la lista de alumnos matriculados ese año. Madrid, 21. 2 h. Minuta a Pascual Asensio sobre el conducto reglamentario para tramitar las peticiones anteriores. Madrid, 22 de mayo. 1 h. Lista citada. Madrid, 16 de julio. 2 h., f. Oficio de Antonio Sandalio al Secretario de la JPMNCN, de la lista de alumnos aprobados en el Curso de Agricultura. Madrid, 18 de julio. $2 \mathrm{~h}$.

- 1837, enero 15, Madrid.

Oficio de Antonio Sandalio a Pascual Asensio sobre su falta de competencia para variar la hora de las clases del Curso. $2 \mathrm{~h}$. Corre unido: respuesta de este último proponiendo el día 17 del corriente como fecha de apertura del Curso. 2 h. Oficio de Pascual Asensio a Juan de Peñuelas informando sobre los cambios de horario. Madrid, 17. 2 h., f. Borrador a Antonio Sandalio remitiendo los oficios de Pascual Asensio sobre este asunto. Madrid, 19. 1 h. Oficio de Antonio Sandalio al Secretario de la JPMNCN explicando los cambios de horario hechos por Pascual Asensio. Madrid, 27. 4 h. Borrador a Antonio Sandalio comunicándole este mismo asunto. Madrid, 7 de febrero. 1 h. Borrador a Pascual Asensio de la JPMNCN, mostrando el desagrado por estos cambios. Madrid, 11. 1 h. Oficio de Antonio Sandalio al Secretario de la JPMNCN explicando los motivos del cambio de horario. Madrid, 22. 4 h. Minuta al Secretario de Estado sobre los pormenores del cambio y los procedimientos seguidos en el trámite. $6 \mathrm{~h}$. Oficio de Antonio Sandalio al Secretario de la JPMNCN, con la contestación de Pascual Asensio sobre el cambio y que acceda a la petición del hijo de Salvador Duchen y su viuda. Madrid, 14 de marzo. $2 \mathrm{~h}$.

- 1837, julio 11, Madrid.

Oficio de remisión de Antonio Sandalio al Secretario de la J.P.M.C.N. de la lista de alumnos aprobados en el curso. 2 h. Se adjunta la citada lista firmada por Pascual Asensio. Madrid, 10. 1 h., f.

- 1837 , noviembre 12, Toledo.

Expediente de Esteban Ledesma sobre la solicitud de plaza de Catedrático de Agricultura de esa ciudad. 3 h., f.

- 1838, julio 26, Madrid.

Borrador al Ministro de Gobernación sobre la conclusión del curso de Agricultura de ese año y remitiéndole la lista de alumnos aprobados. 1 h. Acompaña el borrador de notificación al Presidente de la Junta Gubernativa de este mismo asunto. $1 \mathrm{~h}$. 


\section{JOSÉ LUIS MALDONADO POLO}

- 1840, enero 7, Madrid.

Nota de Pascual Asensio con la fecha del comienzo del Curso. $1 \mathrm{~h}$.

- 1840, enero 10, Madrid.

Borradores al Presidente de la Dirección General de Estudios indicando el comienzo del Curso. $3 \mathrm{~h}$.

$-1843$.

Borrador de justificantes a favor de Camilo de Azpiaza de asistencia al Curso de Agricultura. 1 h.

- 1848, febrero 13. Madrid.

Notificación de Mariano de la Paz Graells a Pascual Asensio remitiendo un memorial de varios alumnos que solicitan cambio de hora de las clases. $1 \mathrm{~h}$. Corre unido: oficio de Pascual Asensio a M. P. Graells exponiendo las causas del cambio. Madrid, 14. 2 h. Oficio de M.P. Graells al Rector de la Universidad de Madrid reproduciendo la contestación de P. Asensio sobre el cambio. Madrid, 17.2 h.

- 1854, enero 20, Madrid.

Oficio de Pascual Asensio a M.P. Graells contando la decisión el Ministro de Fomento sobre la aprobación del presupuesto para el Curso teórico-Práctico de Agricultura en el Real Jardín Botánico para la enseñanza de escolapios y otros eclesiásticos y los procedimientos que deben disponerse en este cometido. $2 \mathrm{~h}$. Se incluye el oficio del Subsecretario del Ministerio de Gracia y Justicia al Director del MNCN, comentando este mismo asunto. Madrid, 31 de marzo. $2 \mathrm{~h}$.

- 1858, noviembre 14, Madrid.

Oficio de Pascual Asensio a M.P. Graells comentando la decisión de la reina sobre que el Tribunal de exámenes lo formen con profesores que hayan impartido las clases y las fechas de celebración. 2 h. Acompaña el oficio de Pascual Asensio a M.P. Graells proponiendo las fechas de exámenes. Madrid, 16. 2 h. 